\title{
Using the Frailty Assessment for Care Planning Tool (FACT) to screen elderly chronic kidney disease patients for frailty: the nurse experience
}

This article was published in the following Dove Press journal:

Clinical Interventions in Aging

\section{Heather Moffatt' \\ Paige Moorhouse ${ }^{1,2}$ \\ Laurie Mallery ${ }^{1,2}$ \\ David Landry' \\ Karthik Tennankore ${ }^{2}$}

'Nova Scotia Health Authority, Halifax, NS, Canada; ${ }^{2}$ Dalhousie University, Halifax, NS, Canada
Correspondence: Paige Moorhouse Division of Geriatric Medicine, Nova Scotia Health Authority, Rm 3323 Veterans' Memorial Building, 5595 Veteran's Memorial Lane, Halifax, NS B3H2EI, Canada

Tel + I 9024734995

Email paige.moorhouse@nshealth.ca
Purpose: Recent evidence supports the prognostic significance of frailty for functional decline and poor health outcomes in patients with chronic kidney disease. Yet, despite the development of clinical tools to screen for frailty, little is known about the experiential impact of screening for frailty in this setting. The Frailty Assessment for Care Planning Tool (FACT) evaluates frailty across 4 domains: mobility, function, social circumstances, and cognition. The purpose of this qualitative study was as follows: 1) explore the nurse experience of screening for frailty using the FACT tool in a specialized outpatient renal clinic; 2) determine how, if at all, provider perceptions of frailty changed after implementation of the frailty screening tool; and 3) determine the perceived factors that influence uptake and administration of the FACT screening tool in a specialized clinical setting.

Methods: A semi-structured interview of 5 nurses from the Nova Scotia Health Authority, Central Zone Renal Clinic was conducted. A grounded theory approach was used to generate thematic categories and analysis models.

Results: Four primary themes emerged in the data analysis: "we were skeptical", "we made it work", "we learned how", and "we understand". As the renal nurses gained a sense of confidence in their ability to implement the FACT tool, initial barriers to implementation were attenuated. Implementation factors - such as realistic goals, clear guidelines, and ongoing training - were important factors for successful uptake of the frailty screening initiative.

Conclusion: Nurse participants reported an overall positive experience using the FACT method to screen for frailty and indicated that their understanding of the multiple dimensions and subtleties of "frailty" were enhanced. Future nurse-led FACT screening initiatives should incorporate those factors identified as being integral to program success: realistic goals, clear guidelines, and ongoing training. Adopting the evaluation of frailty as a priority within clinical departments will encourage sustainability.

Keywords: frailty, screening, feasibility, qualitative, end stage renal disease, decision making

\section{Introduction}

Frailty may be conceptualized as "a clinically recognizable state of increased vulnerability that results from age-associated decline in reserve and function across multiple physiologic systems, such that the ability to cope with acute stressors is comprised". ${ }^{1}$ Multiple studies detail the increased risk of poor outcomes associated with frailty, ${ }^{2-5}$ therefore, the risk to benefit trade-off of standard treatment options (including renal replacement therapies) may need to be reframed when frailty is present.

Patients with chronic kidney disease (CKD) are at increased risk of developing physical dysfunction, cognitive impairment, and frailty. ${ }^{6}$ With an aging population, 
the increasing prevalence of frailty has shifted treatment perspectives for CKD. ${ }^{7-10}$ As a result, patients with CKD and frailty often face complex decisions with regard to treatment options.

Routine identification of frailty offers opportunities to better understand the impact of frailty on outcomes that matter to patients and informs the medical decision-making process at the frontline of care.$^{11}$ Furthermore, because cognitive impairment is a key driver of frailty, the identification of memory loss has implications for how patients and providers approach complex medical decisions or implement complex treatment regimens. ${ }^{12}$ In this situation, further involvement of family is necessary to ensure understanding of and compliance with treatments.

The Palliative and Therapeutic Harmonization (PATH) process was developed to meet the growing need for standardized, evidence-informed guided decision-making in frailty.$^{13} \mathrm{PATH}$ attempts to address the gap in care affecting frail older adults who are not actively dying but whose precarious health situations limit the effectiveness of targeted treatments and interventions. Using comprehensive assessment tools to map out appropriate care options based on frailty burden and patient values, PATH empowers patients and their families to make informed decisions that promote optimal outcomes.

As a first step, PATH relies on the Frailty Assessment for Care Planning Tool (FACT), ${ }^{14,15}$ a practical and interpretable frailty screening tool for non-geriatricians to identify which patients are appropriate for full PATH assessment. The FACT was developed to overcome common barriers to frailty identification and uses the descriptors within the Clinical Frailty Scale (CFS) ${ }^{16}$ Distinguishing features of the FACT method include its focus on collateral report and the inclusion of validated cognitive screening tests (Table 1). Benefits to its use include the ability to pinpoint the area acting as a frailty driver; the refined ordinal frailty scaling approach is divided into frailty's four key domains; mobility, social situation, function, and cognition.

A recent study ${ }^{17}$ compared the reliability of the FACT method with the CFS (which relies on clinician gestalt judgment) using the Frailty Index as the gold standard measure. ${ }^{18}$ Compared to the CFS, the FACT correlated better with the Frailty Index (Pearson $r=0.72$ for the FACT versus $r=0.56$ for the CFS). However, unlike the Frailty Index, the FACT has the advantage of identifying the clinical drivers of frailty for each patient, which can be of value for guided decision-making. The FACT method was therefore selected for this qualitative study of screening for frailty in the nephrology clinic.
Table I Frailty Assessment for Care Planning Tool ${ }^{\mathrm{a}}$ and the Clinical Frailty Scale ${ }^{\mathrm{b}}$

\begin{tabular}{|c|c|}
\hline Modification & Rationale \\
\hline $\begin{array}{l}\text { Separates the original } \\
\text { ordinal scale into }\end{array}$ & $\begin{array}{l}\text { - Allows for easier determination of scale } \\
\text { score when one domain is driving frailty }\end{array}$ \\
\hline 4 domains & $\begin{array}{l}\text { - Suggests areas of focus for further } \\
\text { assessment }\end{array}$ \\
\hline $\begin{array}{l}\text { Adds validated screening } \\
\text { tools for cognitive } \\
\text { assessment }\end{array}$ & - Improves objectivity/reliability of score \\
\hline $\begin{array}{l}\text { Relies on collateral history } \\
\text { instead of self-report }\end{array}$ & $\begin{array}{l}\text { - Improves objectivity/reliability of score } \\
\text { - Helps to identify poor patient insight }\end{array}$ \\
\hline $\begin{array}{l}\text { Combines frailty scores } \\
\text { I-3 ("fit"-“"managing } \\
\text { well”) }\end{array}$ & $\begin{array}{l}\text { Easier to administer, without losing } \\
\text { information that is instrumental to } \\
\text { decision-making }\end{array}$ \\
\hline
\end{tabular}

Notes: a Data from Mallery et al. ${ }^{15}$ bData from Rockwood et al. ${ }^{16}$

The purpose of this study was to: 1) explore the nurse experience in administering the FACT in a specialized outpatient renal clinic; 2) determine how, if at all, their perceptions of frailty changed after initiating routine screening; and 3) determine the factors that influence uptake and administration of frailty screening tools in specialized clinical settings.

\section{Methods}

\section{Renal PATH clinic}

To identify and respond to frailty in CKD patients, the Nova Scotia Health Authority, Central Zone Renal Clinic adopted the methodology of the PATH program and created their own iteration called the Renal PATH clinic. If frailty is identified through FACT screening, CKD patients are referred to the Renal PATH clinic for further assessment and guided decision-making using the more thorough and standardized PATH assessment of frailty, followed by a semi-structured approach to patient/family communication. Through these steps, Renal PATH enables care providers to carefully consider appropriate treatment modalities and supports patients/families to make choices that best suit their individual frailty and circumstances. ${ }^{13}$

The PATH trained nephrology-based nurse practitioner, who runs the Renal PATH clinic, was identified as a clinical champion and co-investigator.

\section{Participants and settings}

The study was conducted in the Renal Clinic at Dalhousie University. The Renal Clinic services outpatient nephrology referrals within the largest region in Nova Scotia, with a catchment area of 800,000 people. Nurse participants from the Renal Clinic were invited to join the study. Inclusion 
criteria for the study required that participants: 1) were nurses working in the Renal Clinic; 2) were trained to use the FACT screening tool; 3) have participated in the FACT screening from project initiation and throughout the 24 months of the study; and 4) were English speaking. All participants provided written informed consent prior to data collection. The study was approved by the Research Ethics Board of the Nova Scotia Health Authority (File number 1015588).

\section{Nurse participant FACT training}

The FACT ${ }^{14,15}$ is an easy-to-administer frailty screening tool for non-geriatric experts designed to reliably identify and stage frailty in any clinical setting that serves an older patient population. The FACT uses similar staging descriptors to the $\mathrm{CFS} ;{ }^{16}$ however, it relies on collateral reporting and the inclusion of validated cognitive screening tests using the Mini-Cog ${ }^{19}$ and the memory axis of the Brief Cognitive Rating Scale ${ }^{20}$ to establish baseline health status and cognitive capacity. The Mini-Cog tests cognition using the following sequence: the repetition of 3 unrelated words, the ability to draw an analog clock face, and recall of the 3 words following the clock-drawing test. The Brief Cognitive Rating Scale (memory axis) tests the ability to recall current events, the current US president, and the names of children or spouse. Other frailty domains, including mobility, function, and social circumstance scores are assigned according to the caregiver's (collateral) report of the patient's baseline status.

The clinic nurses received two 1-hour sessions describing frailty, its clinical significance, and the FACT method of screening. The PATH program coordinator acted as a liaison support between PATH and the frailty screening initiative; she scheduled routine check-ins with the nurse screening group to gather feedback and answer questions, assisted with troubleshooting, oversaw the data collection and entry, and promoted adherence to initiative guidelines throughout the study period.

\section{Research design}

The study employed a descriptive design and modified constructivist grounded theory to extract, analyze and assess core "themes" emerging from the nurse experience in administering the FACT screening tool. As the leading qualitative method in health science research, grounded theory relies on inductive and deductive methods in a process that builds theory and broad concepts from unstructured qualitative data. ${ }^{21,22}$ Data undergoes constant comparison analysis to root out relationship patterns and overarching categories that lead to a substantive theoretical output. ${ }^{23}$ Grounded theory functions well in the health and medicine setting to capture and understand common experiences and explain variation in a logical manner.

A semi-structured interview format was selected to guide the direction of the focus group discussion. The question probes were developed following a review of the literature and were based on the primary research questions. The probes were not designed to identify relationships in the interview data, but to gain insight into the nurses' opinion and to explore the nature of their experiences. Examples of probe questions used to guide group discussion include: What does screening for frailty mean to you? What was your experience implementing the FACT tool? What did you find helpful about the FACT tool? What did you find challenging about using the FACT tool? Do you have a different understanding of frailty after using the FACT tool? What does screening for frailty mean to you? What advice would you give to other groups considering implementation of the FACT tool? The 24-month long experience of integrating the frailty screen into the Renal Clinic routine was considered from the time of introduction to discussions of future directions.

\section{Data collection and methods of analyses}

The interviews were transcribed verbatim. Data analysis was completed by 3 members of the research team. Using the guidelines for grounded theory analysis, ${ }^{24}$ the transcripts were examined and read line-by-line to identify significant excerpts and passages from the discussion, raw data was broken into discrete parts and sorted under conceptual headings. The research team members identified and uncovered emerging themes and developed an open coding framework for the data. Meaningful statements were used to generate specific codes, which were then used to create a scheme using Atlas.ti 6.2 qualitative data analysis software.

Direct focus group interview quotes, terms, and statements were used to support and personalize the data. Participant quotes are presented between quotation marks. The quotes have been de-identified and edited for grammatical clarity.

\section{Results}

Five nurses (4 registered nurses and 1 licensed practical nurse) took part in the focus group interview process; this sample represents the entire Renal Clinic nursing staff. Participants were all female. All participants had over 10 years of nursing experience.

Four main themes emerged during the data analysis: hesitancy ("we were skeptical"), adaptation ("we made it 
Table 2 Final themes and sub-themes

\begin{tabular}{|c|c|}
\hline Theme & Sub-themes \\
\hline \multirow[t]{5}{*}{ I. We were skeptical (hesitancy) } & a. The unknown \\
\hline & b. The challenges \\
\hline & i. Building support \\
\hline & ii. Feasibility \\
\hline & c. Not knowing \\
\hline \multirow[t]{3}{*}{ 2. We made it work (adaptation) } & d. Adapting to change \\
\hline & e. Gaining support \\
\hline & f. Patient/caregiver experience \\
\hline \multirow[t]{4}{*}{ 3. We learned how (development) } & g. Developing approaches \\
\hline & i. Measuring frailty \\
\hline & ii. Implementation \\
\hline & h. Building confidence \\
\hline \multirow[t]{2}{*}{ 4. We understand (internalization) } & i. Recognizing frailty \\
\hline & j. Value added \\
\hline
\end{tabular}

work"), development ("we learned how"), and internalization ("we understand") (Table 2). These themes are described in greater detail in the following text.

\section{Theme I: we were skeptical (hesitancy)}

The Renal Clinic nurse participants described their first reactions to the FACT frailty screening initiative, including the hesitation they originally felt.

\section{The unknown}

The nurse participants invariably described that structured frailty screening was a new approach that had not previously been a component of their clinical training or experience. The goal of the new program, which was to give nurses the ability to identify frailty, was initially considered to be peripheral to the nursing role. Feelings of hesitation arose from the nurses' early inability to anticipate how knowledge of frailty status would impact the clinical care of their patients and because frailty assessment was outside their "comfort zone". Identifying and addressing frailty required a shift in practice that seemed "formal" and "structured", compared to an ad hoc, gestalt approach to frailty ("the eyeball test"), which was a method used by some nurses in the clinic. A common observation was that it was "unusual" to engage patients and caregivers in the manner required by the FACT (ie, objective cognitive testing and collateral report of the patient's situation).

\section{The challenges}

Each nurse participant reflected on the impediments to effective implementation of the frailty screening initiative. Perceived challenges varied with each nurse. Challenges included 1) difficulty gaining support from physicians, and 2) feasibility constraints, especially during implementation; for example, one nurse participant expressed that Renal Clinic team members did not initially understand the purpose of the initiative. Some nurses felt that engaging collateral informants to take part in the FACT screen required too much effort. Despite some gains, certain issues posed ongoing challenges for the Renal Clinic nurses, such as the FACT requirement to obtain input from a collateral historian (such as a family member), which is an important part of the standardized process, as individuals with cognitive impairment tend to overestimate their functional abilities. ${ }^{25}$ Therefore, the Renal Clinic nurses had to routinely encourage collateral historians to accompany patients to their appointments, which was primarily accomplished through phone calls ahead of appointments, with reminders to this effect.

\section{Building support}

We began the process of frailty screening by involving and educating the Renal Clinic nurses about the importance of considering frailty. A central challenge during the initial stages of the project was the nurses' perception that the clinic nephrologists (physicians) were not supportive of the FACT initiative and did not understand the rationale for frailty screening. The nurses noted that most of the clinic physicians were not "engaged" in the process and that they rarely inquired about FACT screening results.

The nurses expressed feelings of concern about the process and questioned the benefit to patients as revealed by the statement:

$[\ldots]$ are we going to put all of our time and effort and energy into this and there's going to be nothing done with it? Is this going to be another one of these 'make work' projects?

Another nurse participant echoed this reaction in the following comment: "If it involves people's time, there has to be some perceived benefit for it, because if not, it's a hard sell". To respond to this concern, the protocol for the initiative included a stipulation that any patient who screened positive for frailty (mild or above) would be referred to the Renal PATH clinic for further assessment in order to take frailty burden into account when making treatment and care planning decisions.

\section{Feasibility}

The issue of time constraint was a concern for all the nurses, especially in the beginning, when they were less accustomed to administering the FACT. All the nurse participants remarked that the time required for FACT administration was 
significantly longer (15 minutes) than what was described during the initial FACT training (6 minutes). Nurse participants described the added responsibility of "fitting it in" into the hectic clinical setting. One nurse participant commented:

$[\ldots]$ time is of the essence $[\ldots]$ the physicians are knocking on the door, they're ready, they don't want any holdups in clinic. So, you're trying to get the assessment done so they can get in there. Or, you need to go in and do it afterwards if you have time.

\section{Not knowing}

Lack of knowledge about frailty, insufficient understanding of the direction of the program, and general uncertainty about the purpose behind the initiative contributed to the development of this theme. The nurses admitted to being unsure about what was to "come" from this program and agreed that they would have benefitted from more direction. Similar to the 'lack of support' detailed above, one nurse participant added, 'I'm going to do this, but what's going to happen with it? Where are we going to go with it [...]?" These concerns were more strongly associated with the early stages of the program, following the initial proposal and their introduction to the FACT initiative.

\section{Theme 2: we made it work (adaptation)}

The second major theme depicts the methods by which the Renal Clinic nurses made accommodations to integrate, gain support for, and implement the FACT frailty screening initiative.

\section{Adapting to change}

The tempo at which the initiative was introduced to the Renal Clinic nurses left little time for second thought. The initiative encouraged the nurses to exercise a collaborative approach and the nurses spoke about the need for behavioral change on a relational level. One nurse participant observed:

We share a lot of information [...] it's nice that you might point out that they [the patient] didn't do well on the frailty [FACT screen] [...] so we'll see if there's anything more to it. That's the nicest thing, we're such a small group, and such small numbers that we can actually communicate and help each other.

Working "cooperatively" and maintaining "flexibility" were interpersonal strategies that were emphasized during implementation. Additionally, the nurse participants discussed the minor modifications to their practice that made application of the FACT easier.

\section{Gaining support}

Throughout the frailty screening initiative, the nurses felt they were compelled to "cheerlead" for the FACT tool, and promote it to clinic staff and physicians. The nurse participants admitted that as they began to appreciate the prevalence and significance of frailty within the Renal Clinic patient population, they became advocates for the continued use of the frailty screen. They perceived their efforts to be largely successful, and reported gaining the support of many of the clinic nephrologists and administrators. Recognizing that, "Some will take more of a keen interest than others".

\section{Patient/caregiver experience}

A sense of commitment to the patients' and caregivers' understanding of the program was of central importance for the Renal Clinic nurses. Nurse participants described taking the time to introduce the screening tool before administering it, and answering questions that arose from the assessment. One nurse participant added that, "I think it depends on whether you've had previous interactions with the patient or caregiver [...] if it's a new patient, it's often more challenging". Discussing the outcomes of the frailty assessment with caregivers, when asked, proved to be a delicate task, though one nurse participant mentioned, "most people aren't surprised" [to learn that the screen identified frailty]. With experience, the nurses developed strategies to appropriately manage these conversations, although they agreed that the approach to conversations about frailty "is not one size fits all”.

\section{Theme 3: we learned how (development)}

The third theme centered on the didactic aspect of the nurses' development, specifically the learned approaches and the selfassuredness that stemmed from their educational gains.

\section{Developing approaches}

Despite the fast pace in the Renal Clinic, the nurses made adjustments to accommodate the FACT screening initiative. Changes in clinic routine were established within the first few months. One nurse participant pointed out:

Once you get comfortable [...] you always kind of do a little meet and greet [...] it's amazing how much information you can gather once you know what to say. But I think we all had to learn that $[\ldots]$. 
The nurse participants explained that they had to modify their dialogue with patients and caregivers to streamline the initiative within their usual care. They regularly exchanged information about scores and outcomes to assist with their own learning and admitted that implementing the FACT was "very different nursing" compared to their customary approach.

\section{Measuring frailty}

Repeatedly, the nurse participants commented that the frailty screening initiative had been an "eye-opening" experience. They recounted several anecdotes about the "unexpected" nature of some of the assessment results, and were surprised by how well the FACT picked up the subtle presence of frailty, which would have possibly been overlooked. After implementing the FACT, one nurse participant commented:

And then you kind of think, oh my God, we've been doing this [standard Renal Clinic nursing assessment] for a long time, what's wrong with me because I'm missing this?

As a result of being given the task of measuring frailty, nurses took ownership of the FACT initiative and learned, through experience, how to better appreciate frailty as an important indicator of health status. One nurse participant said, "I think we've always looked at frailty primarily from a physical point of view". Other nurse participants agreed, adding that they no longer "assume" but now "perform deeper assessments", specifically related to cognition, than they had done previously.

\section{Implementation}

During the course of the frailty screening initiative, the nurses were reticent to ask for clarification on how to use the FACT tool, and frequently referred to their training materials. Improvements in the clarity of the FACT tool were made at the halfway point of the initiative by adjusting the wording of some of the frailty stage definitions. The nurse participants felt that regular updates improved the "uncertainty" of how to implement the FACT.

\section{Building confidence}

As the nurses became more comfortable with frailty screening, they gained confidence in their ability to implement the FACT tool and interpret its results. The high prevalence and degree of frailty detected amongst the Renal Clinic patients aged 75 years and over reinforced their sense of certitude in the process and they began to notice the subtler aspects of frailty. Sixty-seven percent of the population screened were frail: $44 \%$ were mildly frail, $17 \%$ were moderately frail, and $6 \%$ were severely frail. As one nurse participant said:

If we're wondering about a patient, even if they don't meet age inclusion criteria [for screening], we'll often do the FACT along with our assessment so we can quantify their level of frailty.

The nurse participants added that personal growth and self-assuredness were a positive result of the frailty screening work, "in ways that we probably didn't expect it to be, not in measurable ways [...] it just changes your overall perspective". The other nurse participants validated this statement by describing the individual benefits they perceived, and the shared feeling that they each "put their own value on it".

\section{Theme 4: we understand (internalization)}

At the conclusion of the focus group discussion, each nurse participant was asked to reflect on the overall experience of adopting the FACT in their clinic. Data analysis helped to derive the final theme "We understand" from this section of the dialog. From the main theme, 2 primary descriptors emerged: 1) the consideration and recognition of frailty as a crucial determinant of health; and 2) the clear value that FACT brought to their role as healthcare providers.

\section{Recognizing frailty}

During the implementation process, the nurses developed an appreciation for the importance of frailty and identified their earlier "observations" as less informed by comparison. They credited the FACT screening initiative with introducing a unique perspective on frailty, and agreed that their new skillset enhanced their clinical practice. The nurse participants spoke candidly about their shift in thinking, from identifying someone as being "[...] the typical frail person with the cane $[\ldots]$ " to asking "what does that really mean?" or "what does that capture?" in response to FACT descriptors. They spoke about their role as FACT implementers, and of the attentiveness given to nuances and subtle cues presented during administration of the screening tool and subsequent clinic visits. The nurses were quick to act on their observations and to discuss complex cases as a team:

[...] Do they need supports? Do we need to contact social work? Do we need to include family members in the process of medical decision making? Are the offered treatments appropriate in the context of frailty?

In essence, the nurses realized that knowledge of frailty stage can help guide the development of a personalized care plan. 


\section{Value added}

While describing their general perception of the FACT as a systematic approach to evaluating frailty, the nurse participants offered a decidedly positive response and unanimously considered the tool to be of great value. They cited numerous examples of how the FACT improved their expertise as renal nurses and as one nurse participant remarked:

[...] personally, after doing them, I can see the benefit of how I look at the frail patient and their overall care [...]. I find it helps my own assessment, which benefits the patient and their family in the long run.

Another nurse participant described that having the patient's frailty score was "significant" for the overall assessment and care planning of that particular patient. One nurse participant summarized the general feeling of the Renal Clinic nursing staff toward the FACT initiative by saying; "If we feel that using the tool adds value in enhancing patient care, then we will continue to use it".

\section{General perceptions of frailty screening}

The nurse participants were asked to discuss how frailty detection in the Renal Clinic differed before and after the FACT screening initiative. The moderator used the following prompt "What does screening for frailty mean to you?" Some initial descriptors of the FACT included, "structured", "prioritization", "communication", and "training".

Further exploration revealed that the use of a collateral historian, as opposed to the standard of patient self-report, was beneficial for gaining accurate baseline information. The nurse participants added that this approach to frailty screening facilitated a valuable opportunity for interaction between the collateral/caregiver and the nurse. Furthermore, the nurse participants described situations that provoked them to have earnest conversations with family members:

I've had some family members say that it was really an eyeopener for them on how their loved one [...] was a lot more frail, once we put it in those terms, than they thought.

The nurses identified that the standardized language of the FACT led to clear communication among staff regarding the frailty status of their patients. Objective cognitive testing was described as an "interesting" part of the assessment and was weighted heavily as a positive measure for efficiently gauging the cognitive capacity of their patients. They also agreed that having a straightforward protocol for the implementation of the FACT tool allowed them to adjust to the new approach and learn at their own pace, as they were able
Table 3 Perceptions of FACT frailty screening initiative

\begin{tabular}{l|l}
\hline Reasons for FACT screening & $\begin{array}{l}\text { Barriers to FACT } \\
\text { adoption }\end{array}$ \\
\hline Collateral reporting systematic, & Lack of knowledge or \\
standardized approach to identifying frailty & understanding of frailty \\
Objective cognitive testing trained staff & Lack of leadership \\
Confidence in ability to measure frailty & Lack of initiative \\
Organization leadership communication & Lack of supports in \\
and common language & place \\
Frailty as clinical priority supports in place & \\
\hline
\end{tabular}

Abbreviation: FACT, Frailty Assessment for Care Planning Tool.

to refer to the implementation guidelines, as required. This self-directed assimilation period was discussed in further detail and was hypothesized to be the catalyst for a shift in overall perspective:

Our specialty here is kidneys, but this kind of helps us to get an idea of overall health, and how that will kind of intertwine with the health of your kidneys. So that instead of just looking at this one little picture, we're looking at the whole picture.

Despite the challenges, having an organized, standardized approach to screening for frailty was thought to be of significant benefit. The nurse participants agreed that making the frailty screen a clinical priority was imperative to achieve cooperation and teamwork in pursuit of a common goal. The nurse participants also outlined several factors they believed contributed to clinical short-sightedness with respect to frailty, including: "lack of leadership", "lack of knowledge or understanding of frailty", and "lack of initiative". These "cons" were discussed as factors that would work against the successful identification of frailty in a general sense and were not specifically linked to their particular clinic or the FACT tool itself.

During analysis, the content of this portion of the discussion was separated into 2 categories; reasons for FACT frailty screening success (as identified by nurse participants) versus barriers to adoption (see Table 3 for summary of factors).

\section{Discussion}

The renal nurses' experience of the FACT tool was generally a feeling of enhanced efficacy and understanding of frailty, which was directly related to a standardized process of detecting frailty among their patient population and the sense of confidence they felt as a team in being able to provide more appropriate care. Increased knowledge and understanding of frailty was identified by each nurse participant as important and associated with improved decision-making. 
Box I Key factors that contributed to the success of the FACT frailty screening initiative

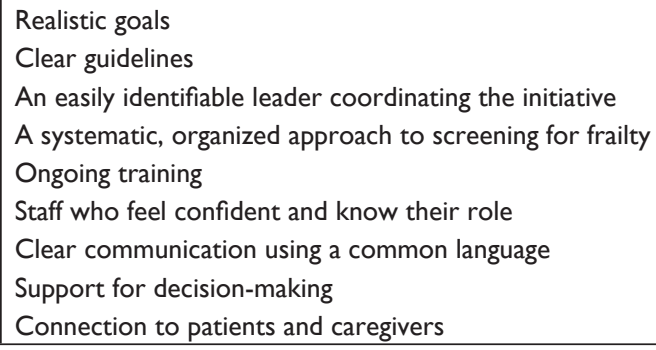

Abbreviation: FACT, Frailty Assessment for Care Planning Tool.

Routine identification of frailty has been effective in other nurse-led screening initiatives. ${ }^{26-28}$ Factors contributing to a positive implementation experience included: realistic goals, clear guidelines, and an easily identifiable leader to coordinate the initiative (Box 1).

The structured implementation of the FACT was cited as a positive factor in executing the project plan, as was ongoing training and support. In contrast to frailty measures, such as the Frailty Index ${ }^{18}$ and the Fried Frailty Score, ${ }^{29}$ the FACT incorporates objective measures of cognitive ability. The nurses appreciated learning how to quickly identify cognitive impairment using the Mini-cog exam ${ }^{19}$ and the Brief Cognitive Rating Scale. ${ }^{20}$ However, nursing staff felt that the impact of the educational component was diminished by limiting the formal training to the pre-rollout phase and would have preferred to have training workshops offered on an ongoing basis throughout the initiative.

In contrast to the numeric output of a frailty index ${ }^{18}$ or tools that use metrics unfamiliar to the patient (grip strength), the FACT tool uses metrics of everyday experience to describe each level of frailty. This is designed to establish a common language between care providers and recipients to facilitate communication. Nurse participants reported more open, informative dialog that communicated the significance of frailty with patients and families. Clear and honest communication enhanced the collaborative practice between the nursing group and the broader Renal Clinic staff by introducing a new lexicon that allowed the healthcare team to discuss frailty using a common language that conveyed frailty stages and the drivers of each stage.

Application of a grounded theory approach allowed the researchers to play an active role in the data collection process and to engage with the nurse participants by conducting interviews and inviting elaboration and clarification related to study phenomena. ${ }^{23}$ Similar studies on nurse experience have also employed grounded theory ${ }^{30}$ epistemology in its modern interpretation, which relaxes strict constructivism and leans more toward interpretative analysis to guide research design. Normalization process theory could also be used to achieve similar outcomes; however; there is less historical evidence of its application in nurse-focused research. ${ }^{31}$

\section{Study limitations}

The most important limitation was the small sample size of the focus group. Because the nurse participants came from the same clinic and shared similar work roles, it is possible that health professionals from disparate clinical settings would have different experiences. Some of the focus group prompts required that the nurse participants provide retrospective data related to the initial stages of the frailty screening initiative. The 2-year interval since program inception raises the possibility of recall bias.

To more fully understand the effects of this program, further research is required to compare these findings with other FACT screening initiatives in similar clinical groups.

The findings in the current study suggest that nurse participants' perceptions of frailty evaluation may be valuable for improving future nurse-led initiatives. For example, future implementation projects could have clearer role definition and identified program champions. Project stakeholders must be identified and have a role in the larger schema. Additionally, while participants did not identify patient or caregiver factors as barriers or facilitators of screening, further study is needed to understand the patient and caregiver experience of FACT screening.

A partnership between the nephrology clinic and the PATH program ${ }^{13}$ was considered positive, as the PATH process was able to help those renal patients in whom frailty was identified. The reciprocal relationship between the 2 programs created a useful system for management of complex cases that required additional resources within the Renal Clinic setting.

Program follow-up noted that adherence to FACT screening initiative guidelines and recruitment of eligible incoming patients had reduced considerably since the time of the interviews. Although the program remains operative, the observed challenges to long-term sustainability are consistent with related literature about the implementation of research programs targeted toward nurse administration, ${ }^{27,28}$ which describe similar issues related to permanency. This disconnect from the experiences captured during data collection was suggested to be due to periods of slow clinic flow over the summer months, as well as the time required to engage the collateral historian during FACT administration being 
difficult to manage amid a hectic schedule. The renal nurses themselves admitted that the initiative had dropped down in terms of clinical priority. It was implied that the nursing staff would welcome more routine contact with research staff to review the findings and to discuss the project trajectory on an ongoing basis. The nurses also expressed a preference for expert professional presence when they required support or clarification related to the FACT screening initiative.

The nurse participants suggested several process items, such as scheduled meetings between program stakeholders nursing, research, and clinician specialists - to review and discuss progress, which might serve as an ongoing motivator. Other suggestions included increased recognition for participating in the process, improved clinic environment, and dedicated time for FACT administration and paperwork.

\section{Conclusion}

This study offers important insights into the nurses' experience with administering the FACT tool to identify frailty in a specialized outpatient renal clinic. Data analysis of a semi-structured focus group discussion with 5 nurse participants identified 4 core themes: initial hesitancy ("we were skeptical"); adaptation ("we made it work"); development ("we learned how"); and internalization ("we understand"). Initial challenges (including a perceived lack of support, uncertainty about benefit, and feasibility constraints) were gradually overcome, while confidence developed as a result of enhanced knowledge about the hitherto underappreciated subtleties and varying dimensions of frailty in older adults. Specifically, nurse participants reported that their understanding of frailty had progressed beyond mere physical indicators ("the typical frail person with the cane") to include more comprehensive (and sometimes less immediately obvious) assessments of frailty domains. Similarly, nurse participants reflected on a greater appreciation of the "big picture" with respect to their patients" health, as opposed to a strict focus on the kidney-related ailments associated with the Renal Clinic.

These results have further implications for clinical programming, particularly for similar nurse-led initiatives. The study indicates that the introduction of a systematic frailty screening tool, implemented under well-defined guidelines, can have success, but must be supervised and, even more importantly, supported to ensure long-term sustainability. Nurse participants consistently noted "lack of support" as a challenge to implementation. As such, clearly assigning the evaluation of frailty as a clinical priority using the FACT might encourage better uptake. Finally, based on experiences identified by the nurse participants themselves (in particular the adaptation and development themes), a focus on training, education, and professional development would likely enhance implementation and prevent the FACT tool from being seen as simply another task, or "make-work project". The nurse participants' positive attitudes stemmed from their ability to incorporate the FACT tool to improve their own interaction with, and understanding of, their patients; rather than merely collecting additional data with no clear practical and immediate benefit.

\section{Acknowledgments}

This initiative was supported by funding from the Nova Scotia Health Authority Research Fund. The authors would like to express gratitude to the Renal Clinic nursing staff for participating in this project and agreeing to an open discussion of their experiences. The FACT tool is copyrighted but is free to access and use online (www.pathclinic.ca).

\section{Disclosure}

Dr Mallery and Dr Moorhouse are the co-creators of the FACT tool and the PATH model of care, and receive consultant fees from institutions implementing the program for delivering a training program for the PATH model of care. The authors report no other conflicts of interest in this work.

\section{References}

1. Xue QL. The frailty syndrome: definition and natural history. Clin Geriatr Med. 2011;27(1):1-15.

2. McAdams-DeMarco MA, Law A, Salter ML, et al. Frailty as a novel predictor of mortality and hospitalization in hemodialysis patients of all ages. J Am Geriatr Soc. 2013;61(6):896-901.

3. Lee DH, Buth KJ, Martin BJ, Yip AM, Hirsh GM. Frail patients are at increased risk for mortality and prolonged institutional care after cardiac surgery. Circulation. 2010;121(8):973-978.

4. Makary MA, Segev DL, Pronovost PJ, et al. Frailty as a predictor of surgical outcomes in older patients. J Am Coll Surg. 2010;210(6):901-908.

5. Clegg A, Young J, Illiffe S, Rikkert MO, Rockwood K. Frailty in elderly people. Lancet. 2013;381(9868):752-762.

6. Anand S, Johansen KL, Tamura M. Aging and chronic kidney disease: the impact on physical function and cognition. J Gerontol A Biol Sci Med Sci. 2014;69(3):315-322.

7. Alston H, Burns A. Ageing renal patients: we need more collaboration between geriatrics services and Nephrology Departments. Healthcare (Basel). 2015;3(4):1075-1085.

8. Johansen KL, Chertow GM, Jin C, Kutner NG. Significance of frailty among dialysis patients. JASN. 2007;18(11):2960-2967.

9. Shlipak MG, Stehman-Breen C, Fried LF, et al. The presence of frailty in elderly persons with chronic renal insufficiency. Am J Kidney Dis. 2004;43(5):861-867.

10. Alfaadhel TA, Soroka SD, Kiberd BA, Landry D, Moorhouse P, Tennankore KK. Frailty and mortality in dialysis: evaluation of a Clinical Frailty Scale. Clin J Am Soc Nephrol. 2015;10(5):832-840.

11. Mallery LH, Moorhouse P. Respecting frailty. J Med Ethics. 2011;37(2): 126-128.

12. Moorhouse P. Care planning in dementia: tips for clinicians. Neurodegener Dis Manag. 2014;4(1):57-66. 
13. Moorhouse P, Mallery LH. Palliative and therapeutic harmonization: a model for appropriate decision making in frail older adults. $J \mathrm{Am}$ Geriatr Soc. 2012;60(12):2326-2332.

14. Frailty Assessment for Care Planning Tool (FACT). 2014. Accreditation Canada. Available from: https://www.accreditation.ca/frailtyassessment-care-planning-tool-fact. Accessed January 1, 2017.

15. Mallery LH, Allen M, Fleming I, et al. Promoting higher blood pressure targets for frail older adults: a consensus guideline from Canada. Cleve Clin J Med. 2014;81(7):427-437.

16. Rockwood K, Song X, MacKnight C, et al. A global clinical measure of fitness and frailty in elderly people. CMAJ. 2005;173(5):489-495.

17. Clark DA, Khan U, Kiberd BA, et al. Frailty in end-stage renal disease comparing patient, caregiver, and clinician perspectives. BMC Nephrol. 2017;18(1):148.

18. Searle SD, Mitnitski A, Gahbauer EA, Gill TM, Rockwood K. A standard procedure for creating a frailty index. BMC Geriatr. 2008;8:24.

19. Borson S, Scanlan J, Brush M, Vitaliano P, Dokmak A. The mini-cog: a cognitive 'vital signs' measure for dementia screening in multi-lingual elderly. Int J Geriatr Psychiatry. 2000;15(11):1021-1027.

20. Reisberg B, Ferris SH. Brief Cognitive Rating Scale (BCRS). Psychopharmacol Bull. 1988;24(4):629-636.

21. Charmaz K. Constructing grounded theory. A Practical Guide Through Qualitative Analysis. Thousand Oaks, CA, USA: Sage Publications; 2006.

22. Rowan M, Huston P. Qualitative research articles: information for authors and peer reviewers. CMAJ. 1997;157(10):1442-1446.

23. Foley G, Timonen V. Using grounded theory method to capture and analyze health care experiences. Health Serv Res. 2015;50(4): 1195-1210.
24. Charmaz K, Belgrave L. Qualitative interviewing and grounded theory analysis. In: J Gubrium, J Holstein, A Marvasti, K McKinney, editors. The SAGE Handbook of Interview Research: The Complexity of the Craft. 2nd ed. Thousand Oaks, CA, USA: SAGE Publications, Inc; 2012 : 347-367.

25. Graham JR, Naglieri JA, editors. Assessment Psychology. Vol. 10. In Weiner IB, editor in chief. Handbook of psychology. Hoboken, NJ, USA: John Wiley and Sons; 2003.

26. Markle-Reid M, Browne G, Gafni A. Nurse-led health promotion interventions improve quality of life in frail older home care clients: lessons learned from three randomized trails in Ontario, Canada. J Eval Clin Pract. 2011;19(1):118-131.

27. Schein C, Gagnon AJ, Chan L, Morin I, Grondines J. The association between specific nurse case management interventions and elder health. J Adv Nurs. 2013;69(10):2262-2273.

28. Kesby SG. Nursing care and collaborative practice. J Clin Nurs. 2002; 11(3):357-366.

29. Fried LP, Tangen CM, Walston J, et al; Cardiovascular Health Study Collaborative Research Group. Frailty in older adults: evidence for a phenotype. J Gerontol A Biol Sci Med Sci. 2001;56(3):146-156.

30. Mills J, Bonner A, Francis K. The development of constructivist grounded theory. Int J Qual Methods. 2006;5(1):25-35.

31. May C, Finch T. Implementing, embedding, and integrating practices: an outline of normalization process theory. Sociology. 2009;43(3): 535-554.
Clinical Interventions in Aging

\section{Publish your work in this journal}

Clinical Interventions in Aging is an international, peer-reviewed journal focusing on evidence-based reports on the value or lack thereof of treatments intended to prevent or delay the onset of maladaptive correlates of aging in human beings. This journal is indexed on PubMed Central, MedLine,

\section{Dovepress}

CAS, Scopus and the Elsevier Bibliographic databases. The manuscript management system is completely online and includes a very quick and fair peer-review system, which is all easy to use. Visit http://www.dovepress. com/testimonials.php to read real quotes from published authors. 